\title{
Connecting systems with short and long ranged interactions: local molecular field theory for ionic fluids
}

\author{
Yng-gwei Chen ${ }^{1,2}$, Charanbir Kaur ${ }^{1}$, and John D. Weeks ${ }^{1,3}$ \\ ${ }^{1}$ Institute for Physical Science and Technology and \\ ${ }^{2}$ Department of Physics and \\ ${ }^{3}$ Department of Chemistry and Biochemistry \\ University of Maryland, College Park, Maryland
}

(Dated: 4th September 2018)

\begin{abstract}
Structural and thermodynamic properties of ionic fluids are related to those of a simpler "mimic" system with short ranged intermolecular interactions in a spatially varying effective field by use of Local Molecular Field (LMF) theory, already successfully applied to nonuniform simple fluids. By consistently using the LMF approximation to describe only the slowly varying part of the Coulomb interaction, which we view as arising from a rigid Gaussian charge distribution with an appropriately chosen width $\sigma$, exceptionally accurate results can be found. In this paper we study a uniform system of charged hard spheres in a uniform neutralizing background, where these ideas can be presented in their simplest form. At low densities the LMF theory reduces to a generalized version of the Poisson-Boltzmann approximation, but the predicted structure factor satisfies the exact StillingerLovett moment conditions, and with optimal choice of $\sigma$ the lowest order approximation remains accurate for much stronger couplings. At high density and strong couplings the pair correlation function in the uniform mimic system with short ranged interactions is very similar to that of the full ionic system. A simple analytic formula can then describe the difference in internal energy between the ionic system and the associated mimic system.
\end{abstract}

\section{INTRODUCTION}

In this paper we describe a new theory for the structure and thermodynamics of ionic fluids based on a generalization of the local molecular field (LMF) theory we have successfully applied to nonuniform simple fluids 1.2 .3 .4 .5 .6 A basic step in the LMF theory is the replacement of longer ranged and slowly varying parts of the intermolecular interactions by an appropriately chosen effective single particle potential. The structure and thermodynamics of the resulting reference system with shorter ranged intermolecular interactions in the presence of the effective field is then related to that of the original system of interest.

This strategy seems particularly appropriate for ionic systems since at long distances the Coulomb interaction is weak and very slowly varying, and systems with short ranged interactions are significantly easier to treat by theory or simulations. However Coulomb interactions can be very strong and rapidly varying at short distances. A key question we address is how to divide the Coulomb interaction into "short" and "long" ranged parts so that the LMF theory can give accurate results. Its answer allows for the first controlled use of the LMF approximation, and we find exceptionally accurate results, even better than those found earlier for fluids with short ranged interactions.

Although the most physically interesting applications of these ideas are probably to nonuniform mixtures of size and charge asymmetric ions, in this initial discussion we consider a uniform one component charged hard sphere system (OCCHS) where almost all the ideas in the LMF theory can be seen in their simplest form 7 The OCCHS is made up of (say positively) charged "ions" comprised of hard spheres with a diameter $d \geq 0$ with embedded positive point charges in the presence of a uniform neutralizing background. The only nontrivial correlations are between the positive ions and for most purposes we can think of this as a one component system with very long ranged repulsive interactions. A special case is the one component plasma (OCP) where there is no hard core $(d=0)$. Nothing in the theory makes essential use of the simplifications in the OCCHS. Generalizations to nonuniform and asymmetric models are straightforward in most cases, and equally good results have been found.

\section{LOCAL MOLECULAR FIELD EQUATION}

\section{A. Nonuniform systems}

We first discuss the qualitative ideas leading to the LMF equation. These will be further developed and made more precise in our discussion of the OCCHS. The simplest application of LMF theory relates the structure and thermodynamics of a nonuniform system of interest with a spherically symmetric pair potential $w(r)$ in an external field whose value at any point $\mathbf{r}_{1}$ is $\phi\left(\mathbf{r}_{1}\right)$ to those of a reference system with a shorter ranged pair interaction $u_{0}(r)$ in a renormalized effective field $\phi_{R}\left(\mathbf{r}_{1}\right) . \phi_{R}$ is supposed to be chosen to take account of the averaged effects of the perturbation potential $u_{1}(r)$, where

$$
w(r)=u_{0}(r)+u_{1}(r) .
$$

This separation of the intermolecular interaction $w$ into two parts can be done in an infinite number of ways, and any choice of $u_{1}$ defines a possible associated reference system. However the averaging procedure leading to the 


\section{Potential Separation}

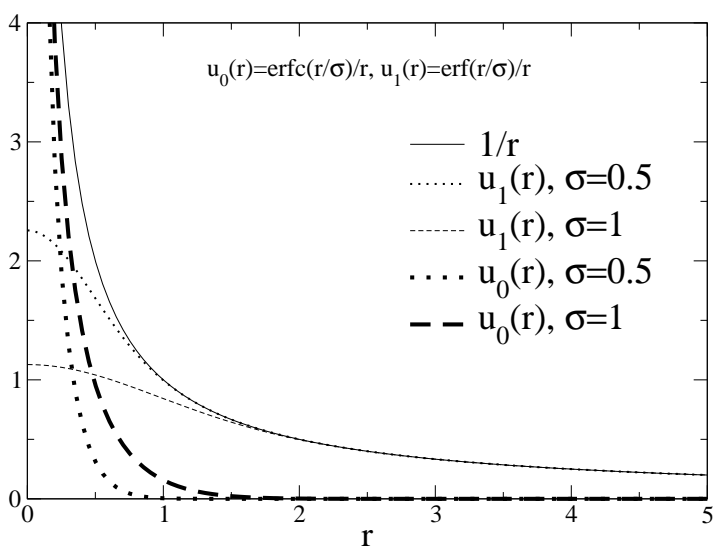

Figure 1: The separation of the $1 / r$ potential into a short ranged piece $u_{0}(r)=\operatorname{erfc}(r / \sigma) / r$ and a long ranged piece $u_{1}(r)=\operatorname{erf}(r / \sigma) / r$. A bigger $\sigma$ corresponds to a longer ranged mimic system $u_{0}(r)$, and a more slowly varying $u_{1}(r)$. Here two relevant $\sigma$ values are shown for comparison.

simple LMF theory can be expected to give very accurate results only for certain properly chosen slowly varying $u_{1}$.

Figure 1 gives examples of separations of the repulsive Coulomb potential we will use in this paper, parameterized by an important length scale $\sigma$. As explained in detail in Section IV when $\sigma$ is chosen larger than some state-dependent minimum size $\sigma_{\min }$, the Coulomb perturbation $u_{1}$ is sufficiently slowly varying that the LMF theory can give very accurate results. This is the crucial step in developing a simple and accurate theory for ionic systems.

We will refer to the resulting special reference systems with properly chosen $\sigma$ as "mimic systems." In realistic models of ionic solutions there are always strong short ranged repulsive core interactions that must be dealt with in any quantitative theory or simulation. The mimic system simply treats the short ranged rapidly varying part of the Coulomb potential as an additional core-like contribution that generates a modified core interaction. As we will see, many properties of the full long ranged system can be very accurately described using those of the short ranged mimic system.

For simple fluids with short ranged interactions the LMF approach has proved most useful when $w$ can be divided into a slowly varying perturbation $u_{1}(r)$ describing the relatively weak and longer ranged attractive interactions and a short ranged rapidly varying core potential $u_{0}$, which accounts for the local excluded volume correlations of the particles $\stackrel{8}{\underline{8}}$ A separation with these qualitative features suffices to motivate the derivation of the basic LMF equation that follows.

For any given $\phi(\mathbf{r})$ an associated $\phi_{R}(\mathbf{r})$ could always found in principle so that the nonuniform singlet density $\rho_{0}\left(\mathbf{r} ;\left[\phi_{R}\right]\right)$ in the reference system (denoted by the subscript 0$)$ equals that in the full system $\rho(\mathbf{r} ;[\phi])$. Of course the latter is not known in advance and its determination is one of the main goals of the theory. However, if a $u_{1}(r)$ can be chosen to be slowly varying over the range of excluded volume correlations induced by the short ranged potential $u_{0}(r)$, then we can make some physically motivated approximations to derive a self-consistent equation to determine the associated $\phi_{R}$.

At high densities we expect that short ranged correlations in both systems are controlled by packing effects from the identical repulsive cores, and it seems plausible that $\phi_{R}$ can be chosen so that both the singlet densities and the conditional singlet densities in the reference and full systems resemble each other. That is, when

$$
\rho_{0}\left(\mathbf{r} ;\left[\phi_{R}\right]\right)=\rho(\mathbf{r} ;[\phi]),
$$

we also expect that

$$
\rho_{0}\left(\mathbf{r}_{1} \mid \mathbf{r}_{2} ;\left[\phi_{R}\right]\right) \simeq \rho\left(\mathbf{r}_{1} \mid \mathbf{r}_{2} ;[\phi]\right)
$$

holds to a good approximation. Here $\rho_{0}\left(\mathbf{r}_{1} \mid \mathbf{r}_{2} ;\left[\phi_{R}\right]\right)$ is the (conditional) density at $\mathbf{r}_{1}$ given that a particle is fixed at $\mathbf{r}_{2}$, directly related to the nonuniform pair correlation function. With this assumption we can derive an equation for $\phi_{R}$ that also turns out to give exact results at very low densities, where pair correlations are not important.

As discussed previously, $\underset{\underline{4}}{\text { by }}$ subtracting the balance of forces as described by the exact Yvon-Born-Green hierarchy ${ }^{7}$ for the full and reference systems we find a relation between the associated forces

$$
-\nabla_{1}\left[\phi_{R}\left(\mathbf{r}_{1}\right)-\phi\left(\mathbf{r}_{1}\right)\right]=-\int d \mathbf{r}_{2} \rho_{0}\left(\mathbf{r}_{2} \mid \mathbf{r}_{1} ;\left[\phi_{R}\right]\right) \nabla_{1} u_{1}\left(r_{12}\right) .
$$

Moreover, if $u_{1}\left(r_{12}\right)$ is very slowly varying over the range of short ranged pair correlations, then $\nabla_{1} u_{1}\left(r_{12}\right)$ essentially vanishes in the range of integration where $\rho_{0}\left(\mathbf{r}_{2} \mid \mathbf{r}_{1} ;\left[\phi_{R}\right]\right)$ differs significantly from $\rho_{0}\left(\mathbf{r}_{2} ;\left[\phi_{R}\right]\right)$ in eq 4. Then we can replace the former by the latter, and take the gradient outside the integral and integrate eq 4

In making this replacement we have ignored correlations between the particles at $\mathbf{r}_{1}$ and $\mathbf{r}_{2}$, and in most contexts this would represent a crude and generally inaccurate approximation. However for slowly varying $u_{1}$ we see that this particular use of the (mean field) approximation can be very accurate, even at high density. Choosing the constant of integration so that the bulk densities in zero field satisfy $\rho^{B}=\rho_{0}^{B}$ we arrive at the simple local molecular field (LMF) equation ${ }^{1.6}$ for the effective field $\phi_{R}$ :

$$
\phi_{R}\left(\mathbf{r}_{1}\right)=\phi\left(\mathbf{r}_{1}\right)+\int d \mathbf{r}_{2}\left[\rho_{0}\left(\mathbf{r}_{2} ;\left[\phi_{R}\right]\right)-\rho_{0}^{B}\right] u_{1}\left(\mathbf{r}_{12}\right),
$$

which is the starting point for our work on nonuniform fluids with both short and long ranged interactions.

To solve this self consistent equation we need to determine the nonuniform density $\rho_{0}\left(\mathbf{r}_{2} ;\left[\phi_{R}\right]\right)$ in the presence of the effective field $\phi_{R}$. The LMF approach does 
not specify or require a particular way to do this. However since the intermolecular interactions and the effective field have shorter ranges in the reference system, both theory and simulations of the nonuniform structure are usually easier to carry out than in the full system. Equation 2 then provides the fundamental link between structure in the nonuniform reference and full systems, and from this thermodynamic functions can be determined.

The name "local molecular field" is suggested by the direct analogy to the spatially varying effective field introduced in the usual mean or molecular field theory for a nonuniform Ising model $\stackrel{9.10}{=}$ However, while the latter theory is usually viewed as a crude approximation, the derivation sketched above suggests that if a proper choice of a slowly-varying $u_{1}$ is made, then accurate results should be found from a self-consistent solution of the LMF equation in many cases, provided that an accurate treatment of the density response $\rho_{0}\left(\mathbf{r} ;\left[\phi_{R}\right]\right)$ to a given $\phi_{R}$ is used. In this paper we use the exact eq 8 below at low densities and results of computer simulations at higher densities, so whatever errors remain arise only from the LMF approximation itself.

\section{B. Uniform systems}

LMF theory is equally useful for uniform fluids $.5 .11 \mathrm{In}$ particular eq $[$ is consistent with the physical idea that in a dense uniform fluid with $\phi=0$ the forces associated with the slowly varying $u_{1}$ from oppositely situated particles essentially cancel in most relevant configurations ${ }^{8.12}$ Moreover, any residual effects are strongly damped by the small compressibility at high density, so we expect that the radial distribution functions will satisfy

$$
g_{0}(r) \simeq g(r),
$$

as predicted by eqs 3 and 5 for $\phi=\phi_{R}=0$. For example, eq [6] holds to a rather good approximation at high density in the uniform Lennard-Jones (LJ) fluid provided that the WCA separation ${ }^{8.13 .14}$ with its relatively slowly varying $u_{1}$ is used, showing the consistency of the physical picture.

This perfect cancellation argument can at best give reasonable results only for uniform fluids at high density. However, LMF theory can be applied to a general nonuniform fluid, and by taking such a perspective and using only the basic eq 2 we can significantly improve on the predictions of eq 6 for pair correlations in uniform fluids 5,11

Corrections to eq [6] can be found by considering the particular external field arising from a fluid particle fixed at the origin, $\phi(\mathbf{r})=w(r)$. The induced density now gives

$$
\rho(r ;[w])=\rho^{B} g(r) .
$$

This exact equation ${ }^{15}$ relating the nonuniform singlet density induced by a fixed particle to the radial distribution function $g(r)$ in the uniform fluid plays a key role in the theory below. There are now net unbalanced forces arising from the fixed particle and eq 5 predicts a nonzero $\phi_{R}$, which can be used in eq 2 to give a more accurate approximation for $\rho(r ;[w])$ and hence $g(r)$.

For the uniform LJ fluid this approach accurately determines the small corrections to eq 6 at high density 5.11 Moreover at very low densities where eq 6 would be very inaccurate, eq[5]gives $\phi_{R}(\mathbf{r})=\phi(r)=w(r)$ and we obtain the exact low density limit? for $g(r)=\exp [-\beta w(r)]$ by using eq 2 and the exact low density limit of the reference system in the field $\phi_{R}$ :

$$
\rho_{0}\left(\mathbf{r} ;\left[\phi_{R}\right]\right)=\rho^{B} e^{-\beta \phi_{R}(\mathbf{r})} .
$$

Here $\beta=\left(k_{B} T\right)^{-1}$.

Similar accurate results for the nonuniform LJ fluid have been found for more general external fields representing hard core solutes of various sizes, for the liquidvapor interface, and for drying transitions $\frac{1,2}{.}$ Thus LMF theory has provided a qualitatively and often quantitatively accurate description of structure, thermodynamics, and phase transitions in fluids with short ranged interactions,$\underline{6}$

\section{ONE COMPONENT CHARGED HARD SPHERES}

In this paper we focus on the uniform OCCHS, where there are $N$ positive ions in a volume $V$ with a uniform neutralizing background that also penetrates the ions. The pair potential $w(r)$ for the ions in the OCCHS is usually written as

$$
w(r)=w_{d}(r)+w_{q}(r),
$$

the sum of a hard sphere potential

$$
w_{d}(r) \equiv\left\{\begin{array}{c}
\infty, r \leq d \\
0, r>d
\end{array}\right.
$$

and the pair potential $w_{q}(r)$ arising from point charges of magnitude $q$, where

$$
w_{q}(r) \equiv \frac{q^{2}}{\epsilon r} .
$$

The separation in eq 9 is a special case of eq 1 and more general separations of $w$ will prove useful in the LMF theory developed below. In eq 11] the solvent is crudely represented by a uniform static dielectric constant $\epsilon$. In the limit $d=0$, the OCCHS reduces to the OCP.

It is convenient to introduce a characteristic length describing the typical distance between neighboring particles. A standard choice is the ion sphere radius a chosen so that

$$
\frac{4 \pi}{3} \rho^{B} a^{3}=1
$$

The nearest neighbor spacing is about $1.6 a$ when the ions are arranged in a simple cubic lattice. 
Thermodynamic properties in the OCCHS can then be characterized using two dimensionless parameters, the ionic strength

$$
\Gamma \equiv \frac{\beta q^{2}}{\epsilon a},
$$

which compares the bare Coulomb interaction energy between two ions separated by the characteristic distance $a$ to $k_{B} T$, and the hard sphere packing fraction

$$
\eta \equiv \pi \rho^{B} d^{3} / 6
$$

Note that $d / a=2 \eta^{1 / 3}$. In the OCP $d=\eta=0$ and thermodynamic properties depend only on the single dimensionless parameter $\Gamma$.

Pair correlations between the ions in the uniform fluid are most conveniently described in terms of the density change induced by fixing a particle at the origin:

$$
\Delta \rho(r ;[w]) \equiv \rho(r ;[w])-\rho^{B}=\rho^{B} h(r),
$$

where $\rho^{B}=N / V$ and $h(r) \equiv g(r)-1$ is the pair correlation function in the uniform fluid.

The unique consequences of the long ranged interaction in the OCCHS are most easily seen by taking the Fourier transform of eq 15] and defining the dimensionless structure factor

$$
S(k) \equiv 1+\Delta \hat{\rho}(k ;[w])=1+\rho^{B} \hat{h}(k),
$$

where the caret denotes a Fourier transform. As argued generally in the seminal work of Stillinger and Lovett ${ }^{16}$ (SL), there should be complete screening at long wavelengths of any induced charge distribution in a conducting ionic fluid. This constrains the behavior at small wavevectors of the charge-charge correlation function. For the OCCHS the only nontrivial correlations are between the positive ions and the results of SL reduce to the requirement that $S(k)$ has the universal form

$$
S(k)=0+k^{2} / k_{D}^{2}+\mathrm{O}\left(k^{4}\right)
$$

independent of any details of the short ranged core potential $w_{d}$ or any other short ranged interactions that might exist. Here $k_{D}$ is the Debye wavevector, defined by

$$
k_{D}^{2} \equiv 4 \pi \beta q^{2} \rho^{B} / \epsilon=3 \Gamma / a^{2} .
$$

The exact vanishing of $S(k)$ at $k=0$ arises from electrical neutrality (the "zeroth" moment condition) and the fixed coefficient of the quadratic term is an example of the famous SL second moment condition. 16,17 This behavior is distinctly different than that found in any fluid with short ranged interactions, where $S_{0}(k)$ at $k=0$ is finite, proportional to the compressibility, and the coefficient of $k^{2}$ depends on the details of the intermolecular interactions.

Thermodynamic properties can be found by integration of the correlation functions. In particular, accounting for the background by taking the appropriate limits of the standard result for a two component system, 18 the excess internal energy (over the ideal gas) of the uniform OCCHS can be exactly written as

$$
\frac{\beta E^{e x}}{N}=\frac{\beta \rho^{B}}{2} \int d \mathbf{r} \frac{q^{2}}{\epsilon r} h(r) .
$$

\section{LMF THEORY FOR OCCHS}

\section{A. Gaussian charge distribution}

We want to use the general LMF equation to describe the ion correlation functions in the uniform OCCHS. It is clear from the derivation in section II that a proper separation of the interaction potential $w=u_{0}+u_{1}$ is required for this self-consistent approach to be accurate. At first glance it may seem natural to use the separation on the right side of eq 9] where $u_{0}$ is taken to be the hard core potential $w_{d}$ and $u_{1}$ is the full point charge interaction $w_{q}$ in eq 11] However at short distances outside the hard core the Coulomb potential can be strong and rapidly varying and such interactions cannot be accurately treated by the averaging used in eq 5

Indeed in the OCP with no hard core there are arbitrarily large and rapidly varying interactions as $r \rightarrow 0$. This limit makes it clear that we should try to separate the point charge Coulomb pair interaction $w_{q}(r)$ itself into a slowly varying part $w_{q 1}(r)$, which we will take as a particularly appropriate $u_{1}(r)$ to use in the LMF theory, and then combine the remainder $w_{q 0}(r) \equiv w_{q}(r)-u_{1}(r)$ with $w_{d}(r)$ (and more generally with any other short ranged core interactions that exist) to give the associated $u_{0}(r)$.

This strategy differs from that used in many density functional and integral equation methods, where one first chooses a mathematically convenient or especially simple reference potential $\tilde{u}_{0}(r)$ and then treats the remainder $w(r)-\tilde{u}_{0}(r)$ as a perturbation, taking advantage of the particular form of the reference system in whatever approximate theories are used. However, for Coulomb systems at least, the existing theories often have large and uncontrolled errors with the usual choices of $\tilde{u}_{0}$. We believe our approach of choosing a slowly varying $u_{1}$ for use in the LMF theory offers many conceptual and computational advantages, and it connects directly to similar physically motivated work on fluids with short ranged interactions.

To that end we interpret the $1 / r$ term in $w_{q}$ as the electrostatic potential of a unit point charge in vacuum. The same slowly varying asymptotic behavior would come from any other normalized charge distribution and the "smearing" of the point charge would produce a less rapidly varying potential at small $r$. This suggests using a properly chosen charge distribution to determine $w_{q 1}(r)$ or $u_{1}(r)$.

Consider in particular as in the Ewald sum method 7,19 
a normalized unit Gaussian charge distribution

$$
P_{\sigma}(r)=\pi^{-3 / 2} \sigma^{-3} \exp \left[-(r / \sigma)^{2}\right],
$$

where $\sigma$ indicates the length scale of the smearing. The particular advantages of this choice will soon become apparent. Our use of a Gaussian charge distribution to determine a slowly varying part of the Coulomb pair interaction is simpler than in the Ewald sum method, which considers periodic images of ion configurations with embedded screening (negative) and compensating (positive) Gaussian charge distributions $\underline{19}$

The electrostatic potential $v_{\sigma}(r)$ arising from eq20 satisfies Poisson's equation $\nabla^{2} v_{\sigma}(r)=-4 \pi P_{\sigma}(r)$, which is easily solved by Fourier transform to give

$$
\hat{v}_{\sigma}(k)=\frac{4 \pi}{k^{2}} \exp \left[-\frac{1}{4}(k \sigma)^{2}\right],
$$

or in $r$-space,

$$
v_{\sigma}(r)=\frac{1}{r} \operatorname{erf}(r / \sigma)
$$

where erf is the usual error function ${ }^{19}$ The point charge model corresponds to the limit $\sigma=0$.

Thus we can write

$$
\frac{1}{r}=\frac{1}{r} \operatorname{erfc}(r / \sigma)+\frac{1}{r} \operatorname{erf}(r / \sigma),
$$

and use this identity to extract from the dimensionless Coulomb pair interaction $\beta w_{q}(r)$ a $\sigma$-dependent perturbation piece $\beta u_{1}(r)$ :

$$
\beta u_{1}(r)=\frac{\beta q^{2}}{\epsilon} v_{\sigma}(r)=\frac{\beta q^{2}}{\epsilon r} \operatorname{erf}(r / \sigma) .
$$

This perturbation remains finite as $r \rightarrow 0$, with $\beta u_{1}(0)=$ $2 \pi^{-1 / 2} \beta q^{2} /(\epsilon \sigma)$.

As illustrated in Figure 1 with appropriate choices of $\sigma$ we can produce a very slowly varying $u_{1}(r)$, which from eq 21] also decays very rapidly in $k$-space. As argued in section [II these are the qualitative features that would be most appropriate for a perturbation $u_{1}$ to give accurate results from LMF theory. The choice of $\sigma$ in the Gaussian charge distribution permits a controlled use of the LMF approximation, and as shown below, with proper choices of $\sigma$ the LMF theory can give exceptionally accurate results.

Any particular choice of $\sigma$ in eq 24 then fixes the associated reference system interaction ${ }^{20}$ as

$$
\beta u_{0}(r)=\beta u_{d}(r)+\frac{\beta q^{2}}{\epsilon r} \operatorname{erfc}(r / \sigma) .
$$

The Coulomb part $w_{q 0}(r)$ of the reference interaction decays very rapidly for $r>\sigma$. For large $r$ we have

$$
\beta w_{q 0}(r) \equiv \frac{\beta q^{2}}{\epsilon r} \operatorname{erfc}(r / \sigma) \sim \frac{\beta \sigma q^{2}}{\sqrt{\pi} \epsilon r^{2}} \exp \left[-(r / \sigma)^{2}\right] .
$$

We call the special reference systems that result from optimal choices of $\sigma$ as discussed in section IVD below mimic systems, since at high density the local structure in the uniform mimic system as exhibited in $g_{0}(r)$ very accurately approximates the $g(r)$ of the full system as in eq[6] This again illustrates the consistency and accuracy of the LMF approach when an appropriate mimic system is used.

Equation 24 can also be interpreted physically as the Coulomb energy arising from two ions each with a rigid Gaussian charge distribution, eq 20 with a width $\tilde{\sigma}=$ $\sigma / \sqrt{2}$. More generally, in ionic solutions we can always replace point charges on the ions by rigid charge distributions without changing any physics if we appropriately modify the core interactions as in eq25 This can be very useful because the rapidly varying short ranged parts of the Coulomb interaction can often be more accurately treated by the same specialized methods used for the other strong core interactions, which must be present in any realistic model of ionic solutions.

\section{B. Scaled LMF equation for the OCCHS}

We now apply the general LMF eq 5 to the OCCHS in the special case where the external field $\phi(\mathbf{r})=w(r)$ is that resulting from an ion fixed at the origin, given by eq 9. This choice allows us to describe uniform fluids, as discussed in Section $\llbracket B$ We take advantage of spherical symmetry and use the Gaussian charge separation of $w(r)$ given in eqs 24 and 25.

The LMF eq 5 can be naturally rewritten in terms of the more slowly varying "perturbation part" of the effective field

$$
\phi_{R 1}(r) \equiv \phi_{R}(r)-u_{0}(r) .
$$

If the perfect cancellation argument were exact, then $\phi_{R}(r)=u_{0}(r)$, or $\phi_{R 1}(r)=0$, corresponding to a fixed mimic particle at the origin, and the resulting induced density in the mimic system would be $\Delta \rho_{0}\left(r ;\left[u_{0}\right]\right)=$ $\rho^{B} h_{0}(r)$, with $h_{0}(r)$ the pair correlation function in the uniform mimic system. The LMF equation corrects this approximation by determining a finite short ranged effective field perturbation $\phi_{R 1}(r)$, which we can picture as arising from a modified solute particle at the origin,, 11 that takes better account of the locally averaged effects of the slowly varying interactions $u_{1}$.

Taking Fourier transforms, and introducing for reasons that will soon become apparent a multiplicative parameter $\alpha$ that scales the amplitude of $\hat{\phi}_{R 1}$, a scaled version of the LMF equation can be written as

$$
\beta \rho^{B} \hat{\phi}_{R 1}(k)=\frac{\alpha k_{D}^{2}}{k^{2}} \exp \left[-\frac{1}{4}(k \sigma)^{2}\right] S_{R}(k),
$$

where

$$
S_{R}(k) \equiv 1+\Delta \hat{\rho}_{0}\left(k ;\left[\phi_{R}\right]\right)
$$


For now we simply note that the original unscaled LMF equation has $\alpha=1$, and view $\alpha$ as a parameter at our disposal. A similar scaling of the LMF equation for systems with short ranged interactions was discussed earlier $\underline{\underline{\underline{1}}}$

Before giving quantitative results in sections $[\nabla$ and $\nabla]$ below, let us discuss some qualitative features of a selfconsistent solution of eq28] Such a solution would determine a short ranged effective field, implying a $\hat{\phi}_{R 1}(k)$ that is finite as $k \rightarrow 0$, along with the associated $\Delta \hat{\rho}_{0}\left(k ;\left[\phi_{R}\right]\right)$. By the fundamental assumption of LMF theory in eq 2 the latter is the LMF approximation to the full $\Delta \hat{\rho}(k ;[w])$ in the OCCHS. In particular $S_{R}(k)$ in eq 29 is the LMF approximation to $S(k)$ in eq [16] and when no confusion will result, we will simply write $S(k)$. $S_{R}(k)$ should be carefully distinguished from $S_{0}(k) \equiv 1+\Delta \hat{\rho}_{0}\left(k ;\left[u_{0}\right]\right)$, which equals the structure factor in the uniform mimic system with $\phi_{R 1}=0$.

\section{Choice of $\alpha$}

In order that $\hat{\phi}_{R 1}(k)$ remain finite as $k \rightarrow 0$ in eq 28 it is clear that with any choice of $\alpha$, the associated $S_{R}(0)$ must vanish identically. (In practice it is not easy to ensure this in a self consistent iterative solution of eq28 and we give in the Appendix in eq $\mathrm{A.3}$ an alternate but equivalent version that is numerically more stable.) Thus the LMF theory gives an approximate structure factor $S_{R}(k)$ that always obeys the exact neutrality condition. Its expansion at small $k$ has the general form

$$
S_{R}(k)=0+B(\alpha) k^{2}+\mathrm{O}\left(k^{4}\right)
$$

resembling eq 17 but the coefficient $B(\alpha)$ of $k^{2}$ depends on $\alpha$ and does not necessarily obey the exact second moment condition. However, by substituting eq [30 into eq [28] we see that the exact result $B(\alpha)=k_{D}^{-2}$ is found if $\alpha$ is chosen self consistently so that ${ }^{21}$

$$
\beta \rho^{B} \hat{\phi}_{R 1}(0)=\alpha .
$$

Thus by proper choice of $\alpha$, we can guarantee that the approximate structure factor $S_{R}(k)$ also obeys the exact second moment condition. We show below in section $\nabla$ that with optimal choices of the key parameter $\sigma$, even the unscaled LMF theory with $\alpha=1$ often gives very good numerical results. However it is conceptually important to realize that the LMF approach can be naturally generalized as in eq 28] so that the exact second moment condition is satisfied, and this adds essentially no numerical costs to the self consistent solution. We use eq 28 along with eq 31 in most of the work reported below, and usually refer to this generalized approach as the LMF theory. If we want to emphasize that the second moment condition is satisfied, we will refer to the LMF2 theory, and distinguish this from the original unscaled version, which we will call the LMF0 theory.

\section{Choice of $\sigma$}

The ability to choose a $\sigma$ larger than some $\sigma_{\min }$ allows for a consistent application of LMF theory to ionic fluids, ensuring that the LMF approximation is used only for slowly varying parts of the Coulomb interactions. The choice of $\sigma_{\min }$ determines an effective Coulomb core size from the core component $w_{q 0}(r)$ of the Coulomb interaction. This may be larger or smaller than the "physical" core size $d$, which can be varied independently in the OCCHS.

For strong coupling with $\Gamma \gg 1$, we expect considerable cancellation of the very strong forces from ions at distances larger than the nearest neighbor spacing $a$. Thus the effective core size $\sigma_{\min } / a$ should be of order unity and essentially independent of $\Gamma$ at large $\Gamma$.

However, for weak couplings with $\Gamma \ll 1$ we would expect that any choice of $\sigma \gtrsim \sigma_{\min }$ with $\sigma_{\min } \simeq \Gamma a$ will be sufficient for the LMF equation to give good results. This (conservative) choice of $\sigma_{\min }$ corresponds to the Bjerrum length. ${ }^{7}$ Only on length scales $\sigma_{\min }$ much less that the average neighbor separation $a$ will even the bare Coulomb interactions between ions exceed $k_{B} T$, which would characterize an effective Coulomb core size. A more detailed argument 22 suggests that we can take even smaller $\sigma$, including $\sigma=0$, for $\Gamma \ll 1$ and still get accurate results from the LMF theory. We will see below that these qualitative considerations hold true generally.

In particular, by choosing $\sigma$ large enough we can guarantee that $\beta \rho^{B} \hat{\phi}_{R 1}(k)$ is nonzero only at small wavevectors, since the Gaussian factor in eq 28 causes rapid decay for $k \sigma \gtrsim 2$. The Gaussian charge distribution produces this very efficient localization of $\beta \rho^{B} \hat{\phi}_{R 1}(k)$ to small wavevectors, and is much superior in this regard to most other smooth distributions.

This property is very important at high density and strong coupling where $S_{R}(k)$ can have significant structure at $k a \simeq 2 \pi$, where $a$ roughly measures the typical distance between nearest neighbor particles. At those larger wavevectors that characterize short ranged structure in $r$-space, $\beta \rho^{B} \hat{\phi}_{R 1}(k)$ essentially vanishes for any choice of $\sigma \geq \sigma_{\min } \simeq a$. Thus for such wavevectors we have $S_{R}(k) \simeq S_{0}(k)$ from a crude linear response argument. Differences in these functions should show up only at small $k$, where $S_{R}(k)$ will satisfy the SL moment conditions while $S_{0}(k)$ remains finite as $k \rightarrow 0$. However at high densities the compressibility in the mimic system is small, so that the absolute differences between $S_{R}(k)$ and $S_{0}(k)$ remain small even at small wavevectors, as will be illustrated in Figure 9 below.

On taking inverse transforms we then expect that $h_{0}(r) \simeq h(r)$ holds true to a very good approximation at high densities, as was qualitatively suggested by the cancellation argument leading to eq [6] Thus we predict a family of uniform mimic systems for different $\sigma \geq$ $\sigma_{\min }$, all of which should give essentially the same short ranged structure at high density that closely approximates that of the full ionic system. This is a dramatic 
example showing that the inverse problem of uniquely determining the intermolecular potential from $h_{0}(r)$ can be ill-conditioned.

A "molecular-sized" choice of $\sigma \simeq \sigma_{\min }$ for the mimic system is considerably smaller than the typical choices made in Ewald sum methods, where $\sigma$ is usually taken to be proportional to the simulation system size ${ }^{19}$ Larger $\sigma$ values will give equally good results, provided that the resulting mimic system is described accurately. However generally there is little to gain from such choices, since the LMF theory is already consistent for $\sigma \simeq \sigma_{\min }$, and it may be more difficult to treat the longer ranged interactions in mimic systems with larger $\sigma$. Thus optimal choices for $\sigma$ are generally near $\sigma_{\min }$.

At lower densities and weak coupling there is little structure in $S_{R}(k)$ and $S_{0}(k)$ at larger wavevectors, and we can choose a much smaller $\sigma_{\min }$ as argued above and still make consistent use of the LMF approximation. Very accurate results for $S_{R}(k)$ are again found from a self-consistent solution of eq 28 but the long wavelength perturbations from $\beta \rho^{B} \hat{\phi}_{R 1}(k)$ are not damped by low compressibility in the mimic system, and $h_{0}(r)$ can differ noticeably from $h(r)$. As $\Gamma \rightarrow 0$, our theory reduces correctly to the exact Debye-Hückel (DH) limit $\underline{7}$

\section{RESULTS AT LOW DENSITY: MIMIC POISSON-BOLTZMANN APPROXIMATION}

At low enough bulk densities, the mimic system's response to $\phi_{R}(r)$ can be described using the simple ideal gas Boltzmann factor as in eq 8 so that

$$
\Delta \rho_{0}\left(\mathbf{r} ;\left[\phi_{R}\right]\right)=\rho^{B}\left[e^{-\beta \phi_{R}(\mathbf{r})}-1\right] .
$$

This also represents the LMF prediction for the full system's $\rho^{B} h(r)$, and requires only that second and higher order virial corrections to the mimic system's pair correlation function can be ignored. When eq 32 is substituted in the LMF equation [28] a closed equation for $\phi_{R}(\mathbf{r})$ results. A self consistent solution is readily found by iteration, using the equivalent but numerically more stable version of the LMF equation in eq A.3.

Equation 32 is the same structural approximation that is used in the Poisson-Boltzmann (PB) theory ${ }^{7}$ Indeed if we set $\alpha=1$ and $\sigma=0$ in the LMF equation 28 combined with eq 32 the results reduce exactly to those of the standard PB theory. The PB theory thus results from taking the full Coulomb interaction of eq [1] as the perturbation $u_{1}$ in the LMF equation and using the Boltzmann approximation for the density response.

We refer to the low density limit of our theory, where eq 32 is used in eq 28 as the mimic Poisson-Boltzmann (MPB) theory. The MPB theory differs from the PB theory only by the choice of $\sigma$ yielding a consistent mimic system along with a choice of $\alpha$ that ensures that the second moment condition is exactly satisfied. As we will see, these simple modifications greatly improve the accuracy and range of validity of the MPB theory.

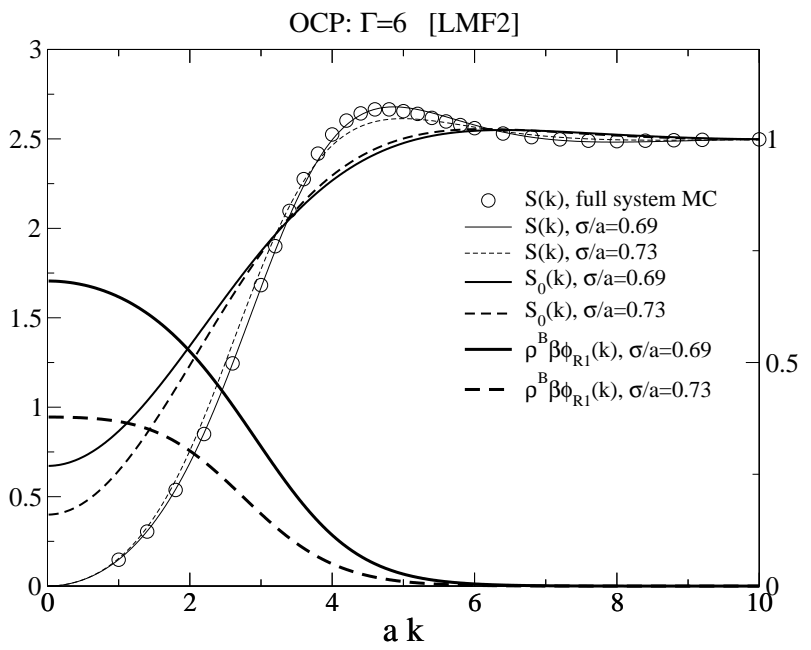

Figure 2: $\quad S_{R}(k)=S(k)$ at moderate coupling for the OCP computed using the MPB theory with different $\sigma$ 's and compared to $S_{0}(k)$ for the uniform mimic system. Also shown are the associated $\rho \beta \hat{\phi}_{R 1}$, which use the scale on the left $y$-axis. When $\rho \beta \hat{\phi}_{R 1}$ is taken into account using the MPB theory, both choices of $\sigma$ give very similar $S(k)$ that compare well with simulation data for the full system. ${ }^{23}$

\section{A. MPB theory for OCP}

Consider first the OCP, where there is no length scale in the potential to suggest an intrinsic core size. A qualitative discussion of the choice of the effective size $\sigma_{\min }$ was given above. In practice it is easy to determine $\sigma_{\min }$ by solving eq 28 using successively larger values of $\sigma$. For $\sigma<\sigma_{\min }$ the results vary strongly with $\sigma$ and are generally very inaccurate. But for all $\sigma>\sigma_{\min }$ the LMF theory is consistent and should give very similar predictions for the full system's structure as exhibited in $S_{R}(k)$, even though the effective fields $\beta \rho^{B} \hat{\phi}_{R 1}(k)$ and the uniform mimic systems' structure factors $S_{0}(k)$ can still vary strongly with $\sigma$. This is illustrated in Figure 2 for the state with $\Gamma=6$, where the convergence of the results for $\sigma / a=0.69$ and $\sigma / a=0.73$ is shown.

The LMF equation itself would continue to give (even more) accurate results for larger $\sigma$ if an accurate theory for the structure $\Delta \rho_{0}\left(\mathbf{r} ;\left[\phi_{R}\right]\right)$ induced by a given $\phi_{R}$ is used. However the simple Boltzmann approximation used in eq[32] for this quantity must fail at higher densities where there are significant correlations between mimic system particles. This sets a $\sigma_{\max }$ above which the results of the MPB theory become inaccurate, very roughly estimated by $\rho^{B} \sigma_{\max }^{3} \lesssim 0.1$ as for hard sphere fluids.

As $\Gamma$ increases, eventually the $\sigma_{\min }$ needed for the accuracy of the LMF approximation exceeds this $\sigma_{\max }$ and the MPB theory fails. The internal consistency or inconsistency as $\sigma$ is varied is very evident from the MPB theory itself. In practice we find very good and consistent results for all $\Gamma \lesssim 6$ and the slight differences in the $S(k)$ curves in Figure 2 and deteriorating results at 

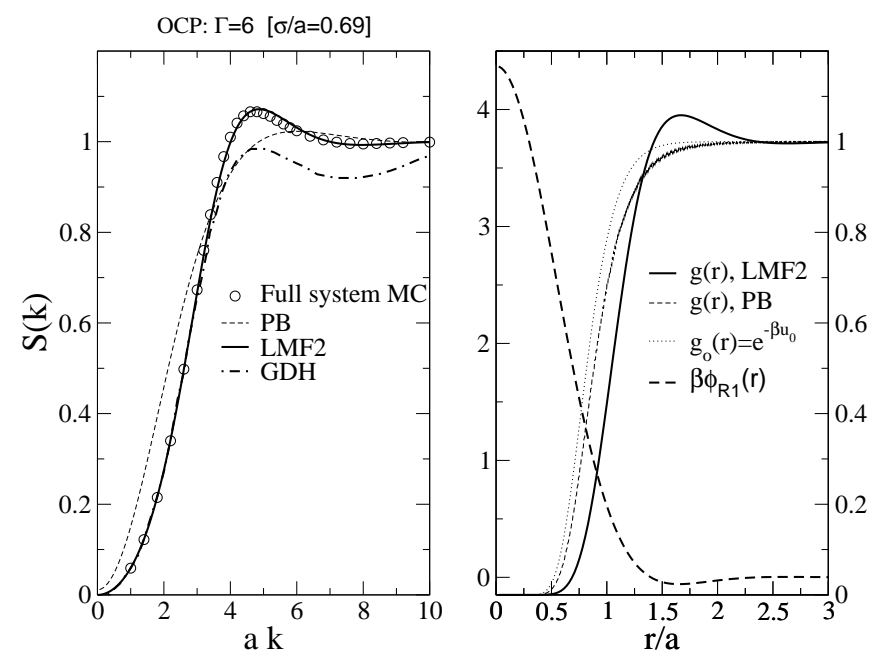

Figure 3: Moderate coupling OCP structure computed using the MPB theory. In the left graph, the LMF2 $S(k)$ is compared with the result of the generalized Debye Hückel theory ${ }^{24}(\mathrm{GDH})$ and the usual PB theory. The right graph makes the same comparison for $g(r)$ and also shows the effective field perturbation $\beta \phi_{R 1}(r)$, which uses the scale on the left $y$-axis. Both the LMF2 and GDH solutions satisfy the second moment condition while PB does not. The GDH result is expressed as an expansion and computed up to its $l=6$ term. ${ }^{24}$

larger $\sigma$ indicate that we are near the upper limit of $\Gamma$ where the MPB theory can be trusted. This represents a surprisingly strong coupling, since the lowest order Boltzmann approximation for the structure in eq 32$]$ is used, and shows the virtues of choosing a mimic system.

As shown in Figure 3 the results of the MPB theory for $\Gamma=6$ are in very good agreement with computer simulations,$\stackrel{23}{ }$ and compare very favorably to those of the usual (nonlinear) PB theory or the generalized Debye-Hückel (GDH) theory developed by Levin and coworkers ${ }^{24}$ Note that the MPB theory, unlike the usual PB approximation, can predict oscillations in both $S(k)$ and $g(r)$ from the self consistent determination of $\phi_{R 1}(r)$ despite using only the lowest structural approximation, eq 32

\section{B. MPB theory for OCCHS}

We now turn to the OCCHS. This has more complex structure because of possible competition between correlations induced by the hard cores and the soft repulsive Coulomb interactions. We follow the usual convention where lengths are measured in terms of the hard core diameter $d$.

In Figure 4 we compare the results of the MPB theory to MC simulations, to results of a density functional treatment, and to the $\mathrm{PB}$ and $\mathrm{DH}$ theories for a low density state with $\eta=0.02$ but with a moderate ionic strength $\Gamma=5.43$. The MPB theory gives excellent re-

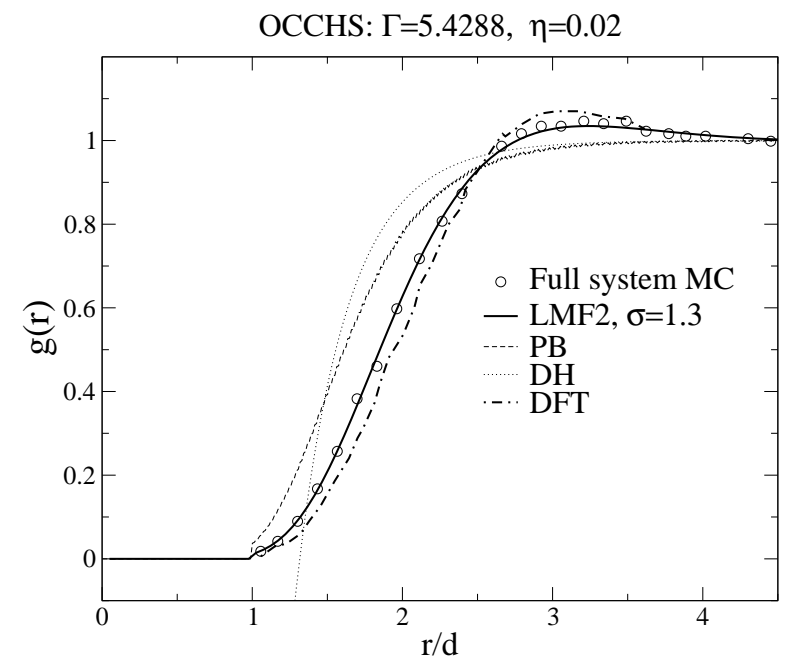

Figure 4: Low density OCCHS structure. The LMF2 $g(r)$ from the MPB theory is compared with the result of density functional theory ${ }^{25}$ (DFT) and the PB and DH approximations. Though constrained to be zero inside the hard core by a boundary condition, the $\mathrm{DH} g(r)$ has a negative region near $r=d$, where $u_{0}(r)$ is also rapidly rising, with a value of -1.62 at contact. The $\mathrm{PB}$ and $\mathrm{DH}$ theories fail to capture qualitatively the onset of oscillation in $g(r)$ at this moderate coupling strength.

sults with a molecular-sized $\sigma=1.3 d$, noticeably better than those of the considerably more complicated density functional theory 25

The right panel in Figure 5 illustrates the role of $\alpha$ for this state, and shows that the self consistent choice of $\alpha=1.2$ can ensure that the exact second moment condition is satisfied, though on the scale of the graph the differences between the $S_{R}(k)$ with $\alpha=1$ are hardly visible. The left panels show that for much weaker coupling with $\Gamma=0.54$ even the usual PB approximation (with $\sigma=0$ ) gives good results, indicating that at weak coupling the choice of $\sigma$ is not so important. However, unlike the MPB theory, the PB approximation can satisfy the second moment condition exactly only in the limit $\Gamma \rightarrow 0$, where it reduces to the $\mathrm{DH}$ approximation $\underline{\underline{7}}$

\section{RESULTS AT HIGH DENSITY AND STRONG COUPLING}

\section{A. Structure in Mimic and Full Systems}

At high densities we expect that the local structure in $r$-space of even the uniform mimic system, where all corrections from $\phi_{R 1}$ are ignored, will closely resemble that of the full system as suggested in eq [6 This is illustrated in Figure [ for the OCCHS for high density states with moderately strong couplings. Canonical Monte Carlo simulations are carried out to obtain the uniform mimic system's correlation function $g_{0}(r), 26$ and these are compared to previous simulations for the full 

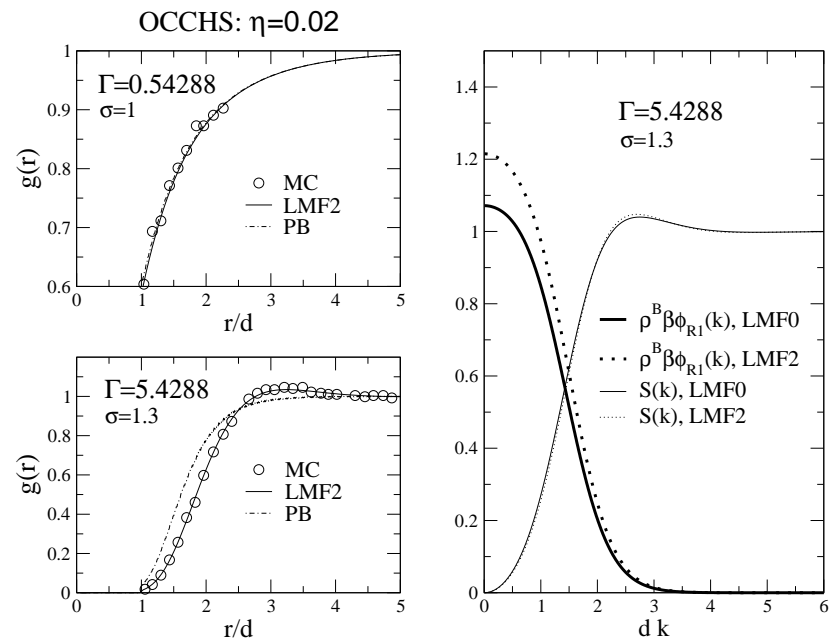

Figure 5: The upper and lower left graphs show the MPB and $\mathrm{PB}$ approximations for the OCCHS $g(r)$ compared to $\mathrm{MC}$ data ${ }^{25}$ for the full system for weak and moderate ionic strengths. The PB approximation is satisfactory only at weak couplings. The right graph shows that varying $\alpha$ so that the second moment condition is satisfied in the MPB theory changes the amplitude of $\beta \rho \hat{\phi}_{R 1}(0)$ but in this case the effects on $S(k)$ are hardly visible on the scale of the graph.

system,, 27 where Ewald sum methods were used to account for the long ranged interactions. Because of the short ranged interactions $u_{0}$ in the mimic system, our simulations are completely straightforward and no Ewald sums or other special treatment of the periodic boundary conditions are required. Again a molecular-sized choice of $\sigma=d$ of order the nearest neighbor spacing suffices.

Also shown on the same graphs are the bare ion potential $\beta w(r)$ and the mimic potential $\beta u_{0}(r)$. Despite the much smaller amplitude of the latter and its much shorter range, the mimic $g_{0}(r)$ has a striking resemblance to the full system's $g(r)$, and the differences are hardly visible on the scale of the graph.

At the strongest couplings, the correlation functions at both densities have a first peak shifted away from contact with the embedded hard sphere. Such a correlation function is very different from the correlation function of a hard sphere fluid, which has its maximum at contact, and shows that the strong short ranged parts of the Coulomb interactions can compete with packing effects from hard cores even at high density. This also emphasizes the importance of having the softer piece $w_{q 0}(r)=q^{2} \operatorname{erfc}(r / \sigma) / \epsilon r$ outside the hard core in our mimic system potential $\beta u_{0}(r)$ in eq 25 in order to reproduce correlation functions in the OCCHS, especially for strong coupling states.

There have been several previous empirical attempts to fit correlation functions for Coulomb systems at high density using effective short ranged systems. The DH limit might suggest that a generalized Yukawa fluid could be useful, 28 but the results were not very accurate and there was no systematic way to determine parameters for
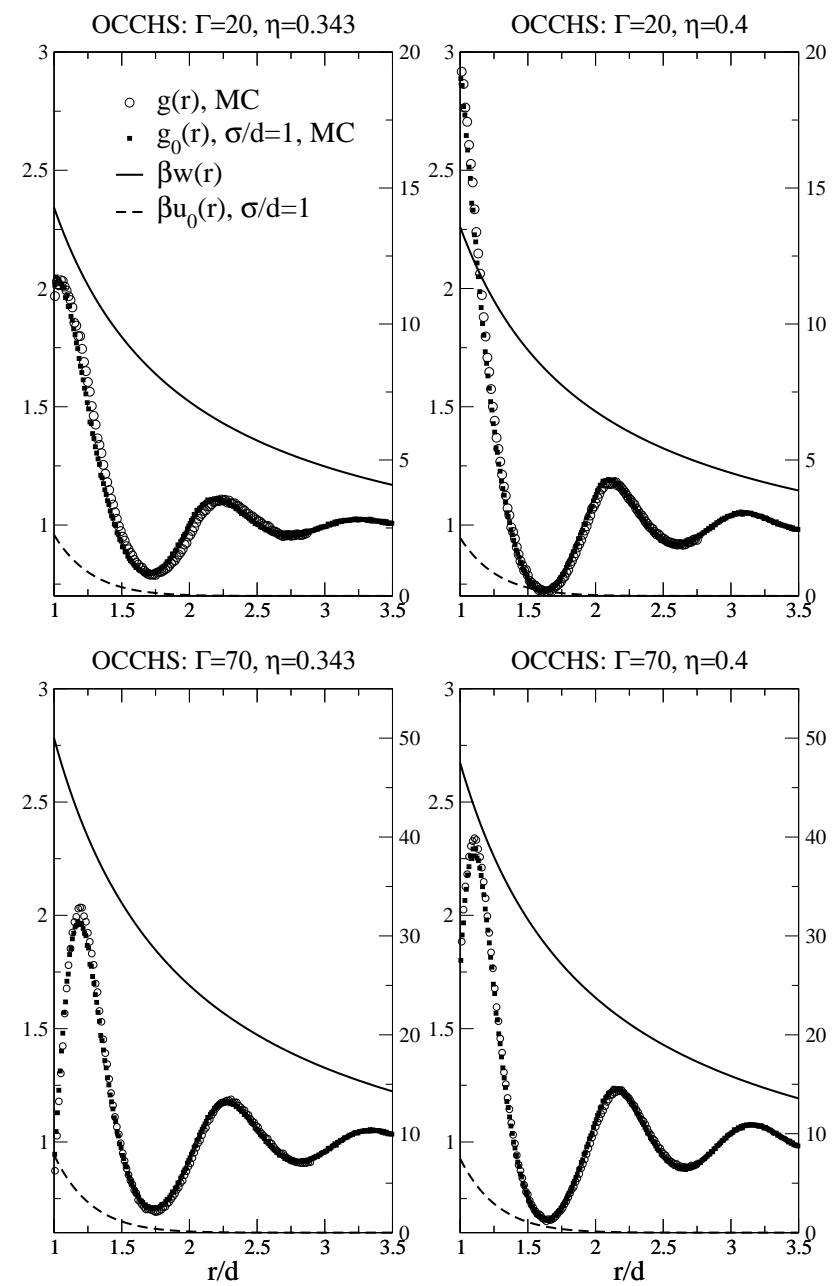

Figure 6: OCCHS correlation functions $g(r)$ at stronger coupling strengths $\Gamma$ and large packing fractions $\eta$ for the full and mimic systems as determined by MC simulations. Note the maximum in $g(r)$ away from contact for $\Gamma=70$, indicating the strength of the Coulomb repulsions. Also shown are $\beta w(r)$, the full potential of the ionic fluid, and the mimic potential $\beta u_{0}(r)$, both of which use the scale on the right $y$-axis. There is a hard core interaction for $r<d$.

the effective potentials.

Most relevant to our work are ion reaction field (RF) methods, where an effective finite-ranged interaction $w_{q 0}^{R F}(r)$ was originally determined from the electrostatic potential of a positive point charge surrounded by a neutralizing uniform spherical charge distribution with a radius $r_{c}{ }^{29}$ Good results for correlation functions were found using the RF method in several applications at high density, though some spurious oscillations were seen near the cutoff $r_{c}$. These were attributed to the discontinuity of the second and higher order derivatives of $w_{q 0}^{R F}(r)$ at $r_{c}$ and better results were found using a smoother "charge cloud" distribution that had discontinuities only in fourth and higher order derivatives at the cutoff 30

Our $w_{q 0}$ in eq 26] can be similarly interpreted in terms 


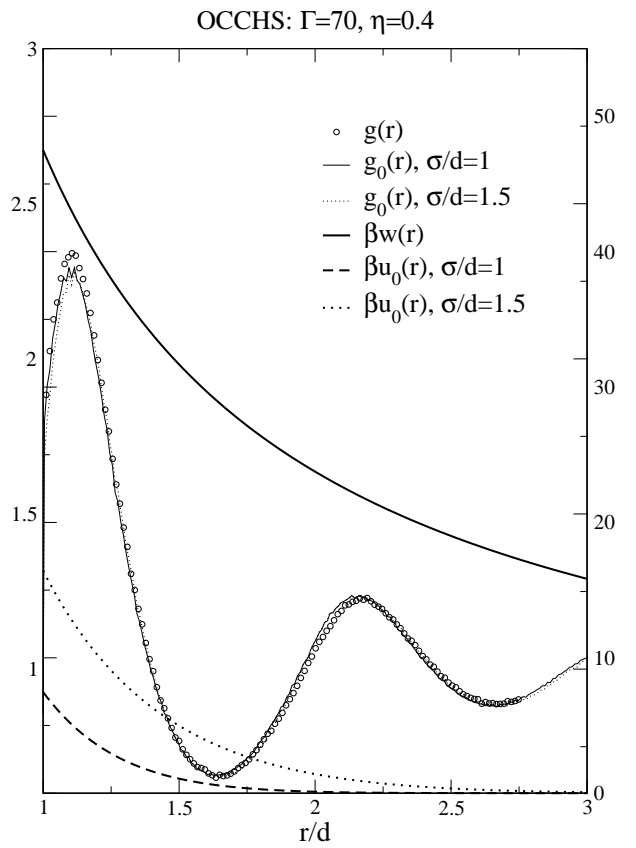

Figure 7: Illustration of mimic system behavior. OCCHS correlation function for the state $\Gamma=70$ and $\eta=0.4$ compared to those of two different mimic systems with different $\sigma$ values. Also shown are $\beta w(r)$, the full potential of the ionic fluid, and the mimic potentials $\beta u_{0}(r)$, all of which use the scale on the right $y$-axis.

of the potential arising from a positive point charge surrounded by a neutralizing Gaussian charge distribution. All derivatives of $w_{q 0}$ are continuous because of the smooth cutoff, and by construction the associated perturbation $w_{q 1}$ decays very rapidly in $k$-space. It is the latter property that fundamentally leads to mimic system behavior with a proper choice of $\sigma$. Our work thus provides a conceptual framework for understanding why RF methods can work as well as they do in some cases and how results can be significantly improved, especially at lower densities or in nonuniform environments by using the LMF theory.

Figure 7 gives a more detailed comparison of the structure of the high density/strong coupling state with $\Gamma=70$ and $\eta=0.4$ to that of two different mimic systems with $\sigma=d$ and $\sigma=1.5 d$. Despite the fact that the (repulsive) potential of the latter is always greater than or equal to that of the former, both mimic systems have very similar correlation functions that agree very well with that of the full system, which can be viewed as the limit $\sigma=\infty$. Thus for high density states all mimic systems with $\sigma>\sigma_{\min }$ have essentially the same short-ranged structure in $r$-space.

Figure 8 gives similar results for the OCP at very strong coupling strengths $\Gamma=80$ and $\Gamma=140$. We find excellent agreement with simulations of the full system ${ }^{31}$ using a mimic system with $\sigma / a=1.4$. As we would expect, for small enough $\sigma$ the good agreement fails, as illustrated by results for $\sigma / a=1$.
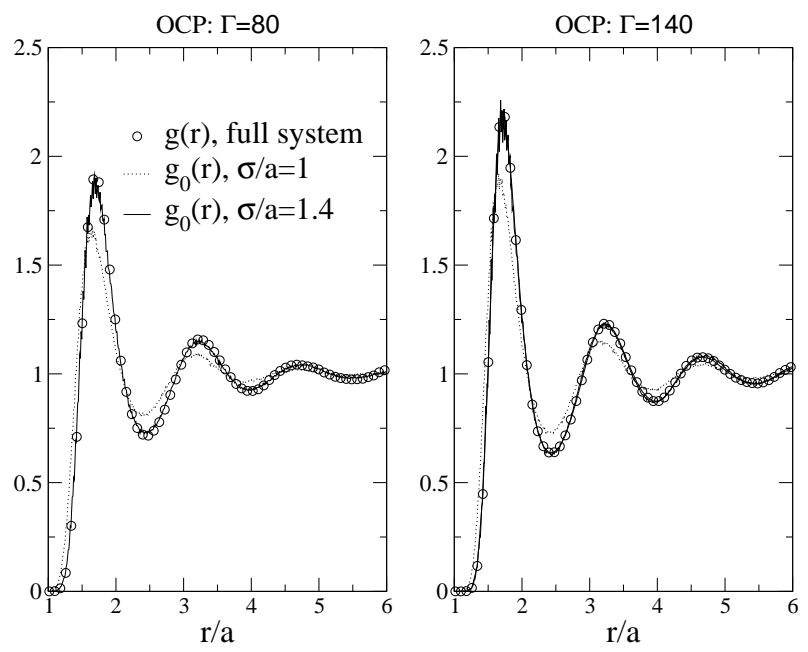

Figure 8: High coupling strength OCP correlations for the full and mimic systems. For $\sigma=1.4 a, g_{0}(r)$ is essentially indistinguishable from the full system's $g(r)$. However a smaller $\sigma=a$ fails to mimic the full system's correlations. Note that around $\Gamma \simeq 170$ the OCP starts to freeze ${ }^{31}$ Our simulations indicate that the mimic system with $\sigma=1.4 a$ also freezes at around the same $\Gamma$.

Figure 9 compares the mimic structure factor $S_{0}(k)$ and a simple estimate for $S_{R}(k)=S(k)$ based on a linear response treatment ${ }^{32}$ of the effects of $\beta \rho^{B} \hat{\phi}_{R 1}(k)$. Only at very small $k$ as revealed in the inset can any differences be seen. The linear response treatment turns out give an $S_{R}(k)$ that satisfies exactly both the zeroth and second moment conditions with $\alpha=1$, and the results converge to $S_{0}(k)$ at larger $k$ controlled by the factor $\exp \left[-\frac{1}{4}(k \sigma)^{2}\right]$ arising from our choice of a mimic system. These features would be found in any more exact treatment and suffice at high densities to give a very accurate estimate of $S_{R}(k)$. This also suggests that the results from other approximate theories may be improved by use of a good mimic system. Indeed, as already shown in Figure [ the simplest possible theory where the effects from $\phi_{R 1}$ are ignored completely, already gives excellent results for short ranged correlations in $r$-space.

\section{B. Internal Energy at High Density}

With accurate approximations for $S(k)$ and $h(r)$ in hand, it is straightforward to calculate thermodynamic properties by integration. The simplest of these is the internal energy, given by eq [19] One can always solve the LMF equation to obtain $h(r)$ and use this integral to compute the internal energy of an ionic system, and we would expect very accurate results. Here we show that because of the great similarity of the local structure of the mimic and full systems in $r$ - and $k$-space at high density, we can obtain an accurate estimate of the energy in terms of the mimic system's energy and a simple analytic correction without explicitly solving the LMF equation. 


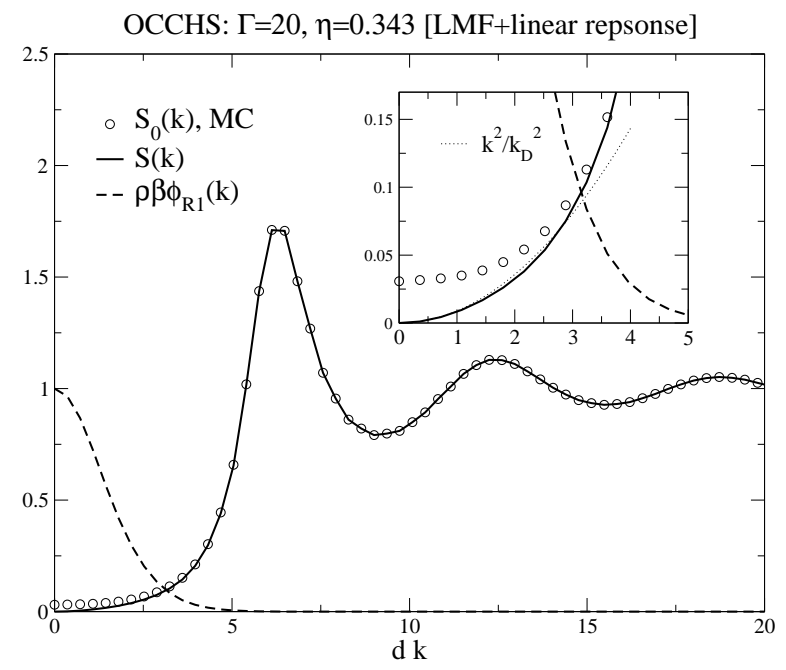

Figure 9: Linear response theory is used to approximate the change in $S_{R}(k)=S(k)$ in the mimic system induced by the the perturbation $\hat{\phi}_{R 1}(k)$. With $\alpha=1$, the linear response theory for $S(k)$ satisfies both SL moment conditions. The inset is a blown-up view of the structure factor at small $k$ where the differences in $S_{0}(k)$ and $S(k)$ can be seen.

Equation [19 can be rewritten in $k$-space and the Coulomb interaction separated into mimic and perturbation parts, so that

$$
\begin{aligned}
\frac{\beta E^{e x}}{N}= & \frac{1}{2} \frac{1}{(2 \pi)^{3}} \int d \mathbf{k} \frac{4 \pi \beta q^{2}}{\epsilon k^{2}}\left[1-e^{-\frac{1}{4}(k \sigma)^{2}}\right] \rho^{B} \hat{h}(k) \\
& +\frac{1}{2} \frac{1}{(2 \pi)^{3}} \int d \mathbf{k} \frac{4 \pi \beta q^{2}}{\epsilon k^{2}} e^{-\frac{1}{4}(k \sigma)^{2}} \rho^{B} \hat{h}(k) .
\end{aligned}
$$

In the first term of eq 33 because $\rho^{B} \hat{h}_{0}(k)$ differs from $\rho^{B} \hat{h}(k)$ only at small $k$, where the factor $1-e^{-\frac{1}{4}(k \sigma)^{2}}$ also approaches zero, we can replace the latter by the former with little error. In the second term, $e^{-\frac{1}{4}(k \sigma)^{2}}$ decays very quickly at higher $k$, so only the small- $k$ features of $\rho^{B} \hat{h}(k) \simeq-1+k^{2} / k_{D}^{2}$ from the SL moment conditions are needed to have an accurate estimate of the integration.

The first term then gives the internal energy of the mimic system

$$
\frac{\beta E_{0}^{e x}}{N}=\frac{1}{2} \int d \mathbf{r} u_{0}(r) \rho^{B} h_{0}(r)
$$

(with a background contribution $-\pi \beta q^{2} \sigma^{2} \rho^{B} / \epsilon$ arising from the use of $h_{0}$ in the integral rather than $g_{0}$ ), while the second term corrects the mimic system's energy and can be integrated analytically to give

$$
\frac{\beta E_{1}^{e x}}{N}=\frac{\beta q^{2}}{\epsilon \sqrt{\pi} \sigma}\left(-1+\frac{2}{\left(k_{D} \sigma\right)^{2}}\right) .
$$

Thus the internal energy can be estimated as

$$
\frac{\beta E^{e x}}{N} \simeq \frac{\beta E_{0}^{e x}}{N}+\frac{\beta E_{1}^{e x}}{N} .
$$

\begin{tabular}{ccccccc}
\hline$\Gamma$ & $\eta$ & $\sigma / a$ & $\sigma / d$ & Full MC & Theory [eq[36] & $\beta E_{0}^{e x} / N$ \\
\hline 20 & - & 1.4 & - & -16.67 & -16.59 & -8.66 \\
20 & 0.343 & 1.4 & 1 & -17.17 & -17.12 & -9.20 \\
20 & 0.4 & 1.4736 & 1 & -17.33 & -17.27 & -9.73 \\
70 & - & 1.4 & - & -60.81 & -60.72 & -32.65 \\
70 & 0.343 & 1.4 & 1 & -61.09 & -61.02 & -32.94 \\
70 & 0.4 & 1.4736 & 1 & -61.32 & -61.24 & -34.56 \\
80 & - & 1.4 & - & -69.69 & -69.64 & -37.54 \\
125 & - & 1.4 & - & -109.73 & -109.74 & -59.50 \\
140 & - & 1.4 & - & -123.09 & -123.13 & -66.85 \\
160 & - & 1.4 & - & $-141.72^{*}$ & $-141.57^{*}$ & -77.23 \\
\hline
\end{tabular}

Table I: Excess internal energy $\beta E^{e x} / N$. The full MC data are taken from references 27 and 31 . At $\Gamma=160$, both the full and the mimic systems are near solidification, and the results depend on initial conditions.

Results for this approximation are compared to $\mathrm{MC}$ results in Table \for a variety of high density/strong coupling states. The Gaussian charge distribution is the key to the accuracy of eq [36, both because of its fast decay in $k$-space and its use in revealing an excellent mimic system for the full ionic system.

Note that at high densities or ionic strengths, the Debye wave vector $k_{D}$ in eq 18 can be very large, making neutrality the major contribution to $\beta E_{1}^{e x} / N \simeq$ $-\beta q^{2} /(\epsilon \sqrt{\pi} \sigma)$. Though the physical interpretation of this term is very different, its limiting value is the same as the self-interaction correction in the Ewald sum method ${ }^{19} \mathrm{~A}$ similar correction was used in ion RF methods ${ }^{29}$

At high density where the compressibility $\chi_{T}$ of the mimic system is very small, we can obtain a good estimate of the internal energy by simply replacing $h(r)$ by $h_{0}(r)$ in eq [19] where because of the background subtraction, a finite result is found for any short ranged $h_{0}(r)$. Separating the Coulomb interaction as in eq 33 and using only the $k=0$ result $\rho^{B} \hat{h}_{0}(0)=-1+k_{B} T \rho \chi_{T}$ in the second integral we find analogous to eq 35.

$$
\frac{\beta E_{1}^{e x}}{N} \simeq \frac{\beta q^{2}}{\epsilon \sqrt{\pi} \sigma}\left(-1+k_{B} T \rho \chi_{T}\right)
$$

which agrees with eq 35 in the relevant limit of large $k_{D}$ and small $\chi_{T}$.

\section{FINAL REMARKS}

It is straightforward to apply these idea to charge and size asymmetric primitive models 33 or to "simple molten salt" models ${ }^{7}$ with softer repulsive cores. We have also used the LMF approximation to look at the OCP near a charged hard wall ${ }^{34}$ Here very accurate results are found both in the weak and strong coupling limits, and the more complicated pair level theory recently introduced 35 is not required. Details will be presented elsewhere, and we only make a few general remarks here. 
We have found for size asymmetric primitive models that the simplest choice of a single $\sigma$ parameter for all species gives excellent results. This can be understood as a consequence of Stillinger and Lovett's fundamental insight that for general ionic mixtures universal consequences of the long ranged Coulomb interactions can be seen in the small wavevector behavior of the chargecharge correlation function. By using the smeared charge distributions implied by a proper choice of $\sigma$, we arrive at a smeared charge-charge correlation function that has significant structure only at small wave vectors. The LMF theory can then reproduce the exact long wavelength behavior found by SL, and the slowly varying smeared Coulomb perturbations have little effect on the shorter wavelength correlations induced by the modified ion cores in the mimic system, just as illustrated here for the OCCHS.

The main complication that arises for such mixtures is that the resulting mimic systems will have short ranged attractive as well as repulsive interactions, but this is required if we want the mimic system's structure to resemble that of the full Coulomb system. However, the LMF theory can then to a very good approximation take care of the "universal" long ranged parts of the Coulomb interactions, which cause major conceptual and computational problems in most approaches, while leaving a nonuniversal, but surprisingly short ranged problem to be treated by whatever means are available. Simulations are straightforward and some recent theoretical developments for treating systems with strong but short ranged interactions look very promising 36 We believe the ideas presented here offer a powerful general perspective, and are actively pursuing their consequences for static and dynamic properties of fluids with both short and long ranged interactions.

\section{ACKNOWLEDGMENTS}

We are grateful to Michael Fisher, Kirill Katsov, Michael Klein, and Lawrence Pratt for helpful comments. We particularly want to thank J. J. Weis for sending us his simulation data for the strong coupling OCCHS correlation functions. This work was supported by the $\mathrm{Na}$ - tional Science Foundation through Grant CHE-0111104.

\section{Appendix: NUMERICALLY STABLE VERSION OF LMF EQUATION}

The LMF equation 28 will produce the desired short ranged $\phi_{R 1}(r)$ with a finite value of $\hat{\phi}_{R 1}(0)$ only if $S_{R}(k)$ rigorously vanishes at $k=0$ as in eq [30] which of course is the exact result. However such self consistent equations are usually solved by iteration and any small errors in an intermediate approximation to $S_{R}(k)$ at small $k$ are greatly amplified. This can lead to numerical instabilities. The following rewriting of eq 28 can alleviate this problem. We can remove the sensitivity at small $k$ by multiplying both sides of eq 28 by $k^{2}$, giving

$$
k^{2} \beta \rho^{B} \hat{\phi}_{R 1}(k)=\alpha k_{D}^{2} \exp \left[-\frac{1}{4}(k \sigma)^{2}\right] S_{R}(k) .
$$

This equation suffices to determine $\hat{\phi}_{R 1}(k)$ everywhere except near $k=0$, where $\hat{\phi}_{R 1}$ is assumed to be regular. We next formally write an identity involving $\hat{\phi}_{R 1}(k)$ that remains finite as $k \rightarrow 0$ :

$$
K^{2} \beta \rho^{B} \hat{\phi}_{R 1}(k)=K^{2} \beta \rho^{B} \hat{\phi}_{R 1}(k),
$$

where $K$ is a (real) constant wavevector. (More generally, we can multiply both sides by a known real function of $k$ that does not vanish as $k \rightarrow 0$.) We now add these two equations as written and divide by $k^{2}+K^{2}$, which yields our final result

$$
\begin{aligned}
\beta \rho^{B} \hat{\phi}_{R 1}(k)= & \frac{\alpha k_{D}^{2}}{k^{2}+K^{2}} \exp \left[-\frac{1}{4}(k \sigma)^{2}\right] S_{R}(k) \\
& +\frac{K^{2}}{k^{2}+K^{2}} \beta \rho^{B} \hat{\phi}_{R 1}(k) .
\end{aligned}
$$

This equation has no divergences at small $k$ and is stable when iterated, with a choice of $K$ of order $k_{D}$. A converged solution will produce a $S_{R}(k)$ that vanishes identically at $k=0$ with $\beta \rho^{B} \hat{\phi}_{R 1}(0)$ finite. From eq 31 this quantity is in fact of order unity when the second moment condition is satisfied. This way of rewriting equations of this kind was suggested to us by Kirill Katsov.
1 Katsov, K.; Weeks, J. D. J. Phys. Chem. B 2002, 106, 8429.

2 Katsov, K.; Weeks, J. D. J. Phys. Chem. B 2001, 105, 6738.

3 Weeks, J. D.; Katsov, K.; Vollmayr, K. Phys. Rev. Lett. 1998, 81, 4400.

${ }^{4}$ Weeks, J. D.; Selinger, R. L. B.; Broughton, J. Q. Phys. Rev. Lett. 1995, 75, 2694.

${ }^{5}$ Weeks, J. D.; Vollmayr, K.; Katsov, K. Physica A 1997, 244,461

6 Weeks, J. D. Annu. Rev. Phys. Chem. 2002, 53, 533.
7 Hansen, J. P.; McDonald, I. R. Theory of Simple Liquids; Academic Press: London, 1986.

8 Weeks, J. D.; Chandler, D.; Andersen, H. C. J. Chem. Phys. 1971, 55, 5422.

9 Binder, K.; Hohenberg, P. C. Phys. Rev. B 1972, 6, 3461.

10 Weeks, J. D.; Gilmer, G. H. J. Chem. Phys. 1975, 63, 3136 .

11 Vollmayr-Lee, K.; Katsov, K.; Weeks, J. D. J. Chem. Phys. 2001, 114, 416.

12 Widom, B. Science 1967, 15\%, 375.

13 Chandler, D.; Weeks, J. D. Phys. Rev. Lett. 1970, 25, 149. 
14 Weeks, J. D.; Chandler, D.; Andersen, H. C. J. Chem. Phys. 1971, 54, 5237.

15 Percus, J. K. Phys. Rev. Lett. 1962, 8, 462.

16 Stillinger, F. H.; Lovett, R. J. Chem. Phys. 1968, 49, 1991.

17 Outhwaite, C. W. Chem. Phys. Lett. 1974, 24, 73.

18 Palmer, R. G.; Weeks, J. D. J. Chem. Phys. 1973, 58, 4171.

19 Frenkel, D.; Smit, B. Understanding Molecular Simulations; Academic Press: San Diego, 2002.

20 This choice of a reference system for the OCP was first suggested in Ceperley, D. M.; Chester, G. V. Phys. Rev. A 1977, 15, 755. However they considered too small $\sigma$ to observe mimic system behavior and used integral equation methods rather than the LMF theory to try to correct for the long ranged interactions.

21 This criterion can equivalently be reexpressed in terms of the normalization of the electrostatic potential ${ }^{17}$

${ }^{22}$ Using the Debye-Hückel approximation for the OCP $S_{R}^{D H}(k)=k^{2} /\left(k^{2}+k_{D}^{2}\right)$ and eq[18]in eq[28 (consistent with $\alpha=1)$ we have $\beta \rho^{B} \hat{\phi}_{R 1}^{D}(k)=3 \Gamma e^{-\frac{1}{4}(k \sigma)^{2}} /\left[(k a)^{2}+3 \Gamma\right]$. The exact $S_{R}(k)$ has the same form at small $k$ (in accord with the SL moment conditions) and generally very different behavior at wavevectors larger than $k_{a} \equiv 1 / a$ that characterize local structure in $r$-space. In order to achieve mimic system behavior with $S_{R}(k) \simeq S_{0}(k)$ at larger wavevectors we require $\beta \rho^{B} \hat{\phi}_{R 1}(k) \ll 1$ for all such wavevectors. Since $S_{R}(k)$ is generally of order unity, it should suffice to choose $\sigma$ so that $\beta \rho^{B} \hat{\phi}_{R 1}^{D}\left(k_{a}\right)=3 \Gamma e^{-\frac{1}{4}(\sigma / a)^{2}} /[1+3 \Gamma] \ll 1$. For large $\Gamma$ we require $\sigma$ of order $a$, but for small $\Gamma \ll 1$ even $\sigma=0$ will still satisfy this rough criterion.

${ }^{23}$ Galam, S.; Hansen, J.-P. Phys. Rev. A 1976, 14816.
24 Tamashiro, M. N.; Levin, Y.; Barbosa, M. C. Physica A 1999, 268, 24.

25 Penfold, R.; Nordholm, S. J. Chem. Phys. 1992, 96, 3102.

26 The $S_{0}(k)$ at larger wavevectors can be accurately determined by a Fourier transform of the simulated $g_{0}(r)$, but at small wavevectors we directly sampled the ensemble average

$$
\frac{1}{N}<\hat{\rho}_{\mathbf{k}} \hat{\rho}_{-\mathbf{k}}>=\frac{1}{N}<\sum_{i \neq j}^{N} \sum e^{-i \mathbf{k} \cdot\left(\mathbf{r}_{i}-\mathbf{r}_{j}\right)}>+1 .
$$

We used $N=250$ and 432 particles for the OCP and up to 686 particles for the high density OCCHS.

27 Hansen, J. P.; Weis, J. J. Mol. Phys. 1977, 33, 1379.

28 Leote de Carvalho, R. J. F.; Evans, R. Mol. Phys. 1997, 92, 211.

29 Hummer, G.; Soumpasis, D. M.; Neumann, M. Mol. Phys. 1992, 77, 769 .

30 Hummer, G.; Soumpasis, D. M.; Neumann, M. J. Phys.: Cond. Matt. 1994, 6, A141.

31 Slattery, W. L.; Doolen, G. D.; DeWitt, H. E. Phys. Rev. A 1980, 21, 2087.

32 See, e.g., Lebowitz, J. L.; Stell, G.; Baer, S. J. Math. Phys. 1965, 5, 1282.

33 Chen, Y.-G.; Weeks, J. D. (unpublished)

${ }^{34}$ Kaur, C.; Chen, Y.-G.; Weeks, J. D. (unpublished)

35 Burak, Y.; Andelman, D.; Orland, H. Phys. Rev. E 2004, 70, 16102.

36 Pratt, L. R.; Ashbaugh, H. S. Phys. Rev. E 2003, 68, 21505. 